\title{
NF-KB-p62-NRF2 survival signaling is associated with high ROR1 expression in chronic lymphocytic leukemia
}

\author{
Elsa Sanchez-Lopez ${ }^{1}$ - Emanuela M. Ghia ${ }^{2}$ - Laura Antonucci ${ }^{1}$ - Natasha Sharma ${ }^{1}$ - Laura Z. Rassenti ${ }^{2}$ Jinyi $\mathrm{Xu}^{3}$. \\ Beicheng Sun ${ }^{4} \cdot$ Thomas J. Kipps $^{2} \cdot$ Michael Karin $^{1}$
}

Received: 18 July 2019 / Revised: 9 January 2020 / Accepted: 10 January 2020 / Published online: 28 January 2020

(c) The Author(s), under exclusive licence to ADMC Associazione Differenziamento e Morte Cellulare 2020

\begin{abstract}
Progression of chronic lymphocytic leukemia (CLL) and resistance to therapy are affected by tumor microenvironmental factors. One such factor is B-cell activating factor (BAFF), a cytokine that is produced mainly by nurse-like cells (NLC) and enhances CLL cells survival and modulates response to therapy. In CLL cells, BAFF activates NF-kB signaling, but how NF- $\mathrm{KB}$ supports CLL survival is not entirely clear. In this study we show that BAFF induces accumulation of the signaling and autophagy adaptor p62/SQSTM1 in a manner dependent on NF- $\mathrm{KB}$ activation. p62 potentiates mTORC1 signaling and activates NRF2, the master regulator of the anti-oxidant response. We found that expression of NRF2 target genes, such as $\mathrm{NAD}(\mathrm{P}) \mathrm{H}$ quinone oxidoreductase 1 (NQO1), is particularly enriched in CLL cells with high ROR1 surface expression $\left(\mathrm{ROR} 1^{\mathrm{Hi}}\right)$. ROR $1^{\mathrm{Hi}} \mathrm{CLL}$ cells with elevated NQO1 expression exhibit resistance to drugs that induce ROS accumulation, such venetoclax. However, such cells are more sensitive to compound $29 \mathrm{~h}$, a pro-drug that only becomes active after being metabolized by NQO1. Accordingly, 29h sensitizes high NQO1 CLL cells to venetoclax. Collectively, our study unravels a previously unknown signaling network through which the NF-kB-p62-NRF2 axis protects ROR1-high CLL cells from ROSinducing therapeutics.
\end{abstract}

These authors contributed equally: Elsa Sanchez-Lopez, Emanuela M. Ghia

\section{Edited by G. Melino}

Supplementary information The online version of this article (https:// doi.org/10.1038/s41418-020-0496-1) contains supplementary material, which is available to authorized users.

Michael Karin

karinoffice@ucsd.edu

1 Laboratory of Gene Regulation and Signal Transduction, Departments of Pharmacology and Pathology, School of Medicine, University of California San Diego, La Jolla, CA 92093, USA

2 Moores Cancer Center, University of California, San Diego, La Jolla, CA 92093, USA

3 State Key Laboratory of Natural Medicines and Department of Medicinal Chemistry, China Pharmaceutical University, Nanjing 210009, China

4 Department of Hepatobiliary Surgery, The Affiliated Drum Tower Hospital of Nanjing University Medical School, Nanjing 210008, China

\section{Introduction}

Chronic lymphocytic leukemia (CLL) is a B-cell malignancy characterized by accumulation of $\mathrm{CD} 19^{+} \mathrm{CD} 5^{+}$ $\mathrm{B}$ cells due to dysregulated cell proliferation and diminished cell death [1]. Components of the tumor microenvironment, including fibroblasts, nurse-like cells (NLC), and T lymphocytes, play key roles in CLL progression, supporting leukemic cell survival and proliferation via cell-cell interactions and secreted factors [1]. Despite advances in CLL treatment, CLL cells are kept in proliferating centers, in which leukemic cells are protected from therapeutic agents. To overcome this inherent drug resistance, it is important to understand how microenvironmental and survival signals regulate this process.

A key microenvironmental factor produced by NLC that control CLL cell survival is B-cell activating factor (BAFF) [2-4]. BAFF, a cell survival and maturation factor for $\mathrm{B}$ cells, can engage through three different receptors, BCMA and TACI, which also bind the related cytokine APRIL; and BAFF-R or BR3. All three receptors are expressed by CLL cells [4]. Blocking BAFF signaling with antibodies that selectively bind the receptors listed 
above reduces CLL viability and increases drug sensitivity $[5,6]$. BAFF downstream signaling involves both canonical and alternative NF- $\mathrm{KB}$ signaling pathways $[7,8]$ and augments CLL survival [9]. Unfortunately, potent and specific inhibitors of $\operatorname{IKK} \beta$, the kinase responsible for canonical NF- $\mathrm{KB}$ signaling, were found to augment inflammation due to enhanced and prolonged NLRP3 inflammasome activation [10]. This had precluded that clinical development of such compounds in CLL and other NF- $\mathrm{KB}-$ dependent lymphoid malignancies. Accordingly, we postulated that a better understanding of the mechanisms and downstream effectors through which NF$\kappa \mathrm{B}$ supports malignant cell survival and treatment resistance may reveal novel targets more amenable for therapeutic intervention.

One important downstream target for NF- $\mathrm{KB}$ that plays a role in several malignancies is p62/SQSTM1, encoded by the SQSTM1 gene [11]. p62 is an autophagy and signaling adaptor that can potentiate mTORC1 signaling [12]. Importantly, by sequestering the negative regulator KEAP1, p62 leads to NRF2 activation, thereby supporting resistance to ROS-inducing drugs [13]. Importantly, NF-kB-mediated p62 induction contributes to termination of NLRP3 inflammasome activation by targeting damaged mitochondria to mitophagic elimination [14]. Consequently, p62 ablation results in the same phenotypic enhancement of NLRP3 inflammasome activation in myeloid cells as achieved by IKK $\beta$ inhibitors [10, 15]. However, p62 mediated regulation of NLRP3 inflammasome activation and mitophagy has only been detected in myeloid cells, such as macrophages, whereas in other cell types, such as hepatocytes, p62 was found to control metabolism and malignancy through its effects on mTORC1 and NRF2 $[16,17]$. Here we examined whether BAFF-dependent activation of NF- $\mathrm{KB}$ induces p62 accumulation and NRF2 activation in CLL and investigated its role in leukemic cell survival and therapy resistance.

\section{Materials and methods}

\section{CLL samples, cell culture, and reagents}

Blood samples were collected from CLL patients at Moores Cancer Center under informed consent (UCSD HRPP\#171884). Peripheral blood mononuclear cells were isolated by density centrifugation with Ficoll-Paque PLUS (GE Healthcare Life Sciences) and suspended in 90\% fetal bovine serum (Omega Scientific, Tarzana, CA, USA) and $10 \%$ dimethyl sulfoxide (Sigma-Aldrich, St. Louis, MO, USA) for viable storage in liquid nitrogen.

Nurse like cells were generated as described [18]. Briefly, $\mathrm{CD} 14^{+}$cells were isolated by positive selection with magnetic beads (Miltenyi Biotec, San Diego, CA, USA) from PBMCs of healthy donors. Then $\mathrm{CD} 14^{+}$cells were seeded in the presence of CLL cells at 1:20, and incubated for 14 days in complete RPMI supplemented with $10 \%$ human serum (Gemini Bioproducts, Sacramento, CA, USA) and $1 \%$ penicillin/streptomycin (Gibco, Thermofisher Scientific Carlsbad, CA, USA).

The MEC1-ROR1 CLL cell line was generated as described to resemble ROR $1^{\mathrm{Hi}}$ CLL cells [19]. These cells were cultured in RPMI 1640 medium (Gibco) with 10\% fetal bovine serum (Gibco) and $1 \%$ penicillin/streptomycin (Gibco) and maintained at $37{ }^{\circ} \mathrm{C}$ in a humidified atmosphere of $5 \% \mathrm{CO}_{2}$.

Human recombinant BAFF was from Tonbo Biosciences (San Diego, CA, USA); BMS-315541, a selective IKK $\beta$ inhibitor [20], was provided by Bristol-Myers Squibb (Irvine, CA, USA). The BCL2-antagonist venetoclax and the mTORC1 inhibitor rapamycin were purchased from Selleckchem (Houston, TX, USA). Compound $29 \mathrm{~h}$ was synthesized by Jinyi Xu [21]. Chloroquine was from Sigma Aldrich (St. Louis, MO, USA) and N-acetyl L-cysteine from Selleckchem.

\section{Flow cytometry}

Flow cytometry was performed as described [22]. Cells were stained for $20 \mathrm{~min}$ using the following antibodies: anti-ROR1 mAb (4A5) conjugated with Alexa-647 (4A5Alexa-647) was generated in Dr. Kipps laboratory. CLL cells were also analyzed for CD5, CD19, CD20, CD23, CD38, and ZAP-70, using monoclonal antibodies (mAbs) conjugated to allophycocyanin (APC), peridininchlorophyll-protein (PerCp), fluorescein isothiocyanate (FITC), or phycoerythrin (PE), respectively (Becton Dickinson, Pharmingen, La Jolla, CA, USA). Fluorochrome-conjugated, isotype control mAbs of irrelevant specificity were used in all experiments to control for nonspecific staining. Data were acquired using a duallaser FACS Calibur (BD Biosciences) and analyzed by FlowJo software (TreeStar, Ashland, OR, USA). Lymphocytes were gated on the basis of their forward-angle light scatter and side-angle light scatter and expression of ROR1 was analyzed on CD19-positive cells. Relative ROR1 expression was calculated by the delta mean fluorescence intensity $(\Delta \mathrm{MFI})$, which is the mean fluorescence intensity of CLL cells stained for ROR1 minus the mean fluorescence intensity of the same cells stained with a nonspecific isotype matched antibody [22]. A $\triangle \mathrm{MFI}$ of 32 was used as threshold to stratify the samples in two groups: low ROR $1<32 \Delta$ MFI $(n=10)$, and high ROR1 $>32 \Delta$ MFI $(n=26)$. The $\Delta$ MFI values of all samples used in the study are shown in Supplemental Table S1. 


\section{Gene expression and gene set enrichment analyses}

We analyzed ROR1 expression in a previously published gene expression data from Microarray Innovations in Leukemia study datasets (GSE13204) of 448 CLL patients [23] deposited in PubMed GEO database. Based on the level of RORI expression, cases with an expression value above the median were designated as ROR $1^{\mathrm{High}}(N=224)$ and cases with RORI expression below the median were designated ROR1 $1^{\text {Low }}(N=224)$.

We used the GSEA software [24] to perform gene-setenrichment analyses (GSEA) of NRF2 inducible genes [25], and mTORC1 upregulated genes [26] on microarray GSE13204 dataset. A gene set was considered significant when the false discovery rate (FDR) was less than $25 \%$ [24]. The gene-set size (SIZE), the enrichment score (ES), the normalized ES (NES), the nominal $p$ value (NOM pval), and the FDR q value (FDR q-val) were determined.

In addition, we segregated GSE13204 dataset into two groups designated as ROR $1^{>90 \%}(N=45)$, representing the $10 \%$ of patients who had CLL cells with the highest RORI mRNA, and as $\operatorname{ROR} 1^{<10 \%}(N=45)$, representing the $10 \%$ of patients with the lowest ROR1 mRNA. Similarly, GSEA of NRF2 inducible genes was performed on microarray data of ROR $1^{>90 \%}$ and ROR $1^{<10 \%}$ cases.

GSEA of RNA-seq data previously deposited in PubMed GEO database (GSE114382) of three CLL samples were collected before therapy (Pre) and at day 28 of Cirmtuzumab treatment (D28) was performed as described [27]. Each D28 sample was collected after patients had received two doses of $16 \mathrm{mg} / \mathrm{kg}$ Cirmtuzumab. Total RNA isolation and RNA-seq data analysis was performed as described [27].

\section{Immunoblot analysis}

Nuclear fractions were prepared using NE-PER ${ }^{\mathrm{TM}}$ Nuclear and Cytoplasmic Extraction kit (Invitrogen, Thermofisher); and whole cell lysates were prepared in NP40 buffer containing protease and a phosphatase inhibitor cocktail. Samples were separated by SDS-PAGE. Proteins were transferred into PVDF membranes, blocked in 5\% BSA and $1 \mathrm{X}$ TBST for $1 \mathrm{~h}$, and incubated overnight at $4{ }^{\circ} \mathrm{C}$ with the following antibodies: phosphorylated p65 (\#3031, Cell signaling, Beverly, MA, USA), HDAC (sc-7872, Santa Cruz Biotechnology, Dallas, TX, USA), GAPDH (sc47724, Santa Cruz Biotechnology), tubulin (T9026, Sigma), p62 (GP62-C, Progen, Long Beach, CA, USA), phosphorylated S6 ribosomal protein (\#2211, Cell Signaling), HSP90 (G10419, BD transduction labs), S6 ribosomal protein (\#2317, Cell Signaling), NQO1 (\#62262, Cell Signaling), SOD1 (sc-11407, Santa Cruz Biotechnology), actin (A4700, Sigma), LC3B (\#2775, Cell Signaling), cleaved PARP (\#5625, Cell Signaling), PARP (sc-8007, Santa Cruz
Biotechnology) and cytochrome C (\#11940, Cell Signaling). Secondary antibodies were added for $1 \mathrm{~h}$. at room temperature and detection was done using Clarity ${ }^{\mathrm{TM}}$ Western ECL Substrate (Biorad).

\section{Reactive oxygen species (ROS) analysis}

CLL cells were seeded on 96 well plates and treated as indicated. Mitochondrial ROS was determined by MitoSox Red Mitochondrial Superoxide indicator (M36008, Invitrogen, Thermo Fisher Scientific), and general oxidative stress by CM-H2DFCDA (C6827, Invitrogen, Thermo Fisher Scientific) as manufacturer's instructions. Fluorescence intensity was determined using TECAN SPARK plate reader.

\section{CRISPR/Cas9 transfections}

Transfections of MEC1-ROR1 cells with p62 or NQO1 CRISPR/Cas9 KO plasmids (Santa Cruz Biotechnology) were done using Lipofectamine 3000 (Thermofisher) according to manufacturer's instructions. Loss of p62 or NQO1 was confirmed by IB.

\section{RNA isolation and quantitative real-time PCR (QPCR)}

RNA was extracted using RNAeasy Mini kit, and cDNA was synthesized using SuperScript VILO cDNA Synthesis Kit (Thermofisher). mRNA expression was determined by QPCR in a CFX96 thermal cycler (Biorad, Hercules, CA, USA) as described [14]. Data are presented in arbitrary units and were calculated by $2^{\wedge}(-$ delta CT) method. Primer sequences were obtained from the NIH qPrimerDepot and provided by Integrated DNA technologies and are shown in Supplemental Table S2.

\section{Cellular viability}

CLL cells or MEC1-ROR1 cells were seeded in 96 well plates and treated as indicated. Cellular viability was determined 24, 36 or $48 \mathrm{hr}$ later using CellTiter-Glo 2.0 reagent (G924B, Promega, San Luis Obispo, CA, USA) accordingly to manufacturer's instructions. Fluorescence intensity was determined using Infinite M200 Pro TECAN plate reader.

\section{Statistical analysis}

Data are shown as mean $\pm \mathrm{SD}$ or mean $\pm \mathrm{SEM}$, as indicated Statistical significance was determined using two-tailed student's $t$-test, and $p$ values lower than 0.05 were considered statistically significant. GraphPad Prism was used for statistical analysis and graphing. 
a

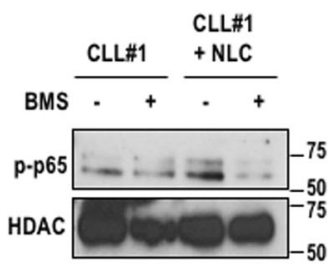

b

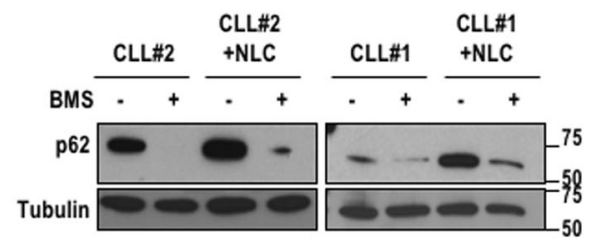

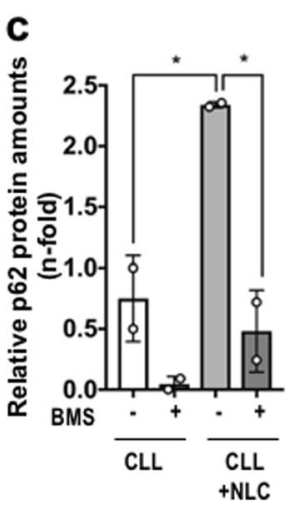

Fig. 1 Nurse-like cells induce p62 accumulation via NF-кB activation in CLL cells. a Primary CLL cells were cultured in the presence or absence of NLC, and incubated with or without $5 \mu \mathrm{M}$ of the IKK $\beta$ inhibitor BMS-345541 (BMS) for $24 \mathrm{hr}$. Nuclear phosphorylated p65 was detected by immunoblotting (IB). b p62 expression in CLL cells treated as described in (a) was examined by IB of whole cell lysates. $\mathbf{c}$ Relative p62 accumulation in CLL cells treated as in (a). $n=2$. $* p<0.05$.

\section{Results}

\section{NLC or BAFF induce NF-kB-mediated p62 accumulation in CLL}

NLC promote CLL cell survival by secreting factors that activate NF- $\mathrm{KB}$ and/or other survival-signaling pathways $[8,28]$. Consistent with our previous findings [7], canonical $\mathrm{NF}-\kappa \mathrm{B}$ signaling was activated in primary CLL B cells cultured with NLC, resulting in nuclear accumulation of phosphorylated p65, which was blocked by the IKK $\beta$ inhibitor BMS-345541 [20] (Fig. 1a). As previously seen in myeloid cells [14], NLC-mediated NF- $\mathrm{\kappa B}$ activation also led to p62 accumulation in an IKK $\beta$-dependent manner (Fig. 1b, c). Of note, some CLL cells, e.g. CLL \#2 in Fig. 1b, exhibited high p62 expression prior to incubation with NLC. But even in such cases the IKK $\beta$ inhibitor led to nearly complete disappearance of p62 (Fig. 1b). One of the major factors through which NLC activate NF- $\mathrm{KB}$ in CLL cell is BAFF [7, 8]. Accordingly, BAFF-induced p65 phosphorylation and p62 accumulation in CLL cells in a manner sensitive to IKK $\beta$ inhibition (Fig. 2a-d). BAFF also induced NF- $\mathrm{\kappa B}$ activation and $\mathrm{p} 62$ accumulation in the CLL cell line MEC1-ROR1 (Fig. S1a, b). Accumulation of p62 occurs when autophagy is impaired [29]. BAFF stimulation induced the lipidation of LC3, indicating stimulation of autophagy in CLL cells (Fig. 2e, f). Together these results indicate that the accumulation of $\mathrm{p} 62$ induced by BAFF is due to increased NF- $\mathrm{\kappa B}$ activation rather than impaired autophagy.

\section{BAFF-induced NF-KB activation activates mTORC1}

p62 interacts with and potentiates activation of the mTORC1 complex, an important metabolic sensor and

regulator [30]. Consistent with p62 induction, BAFF also increased S6 ribosomal protein phosphorylation in most CLL specimens (Fig. 3a, b), suggesting the activation of S6 kinase, a downstream target for mTORC1 [31]. As observed for p62, BAFF-induced S6 phosphorylation was also IKK $\beta$ dependent (Fig. 3a, b). As expected [31], incubation of CLL cells with rapamycin, an mTORC1 inhibitor, blocked the induction of S6 phosphorylation (Fig. 3c). Rapamycin, however, had no effect on p62 expression (Fig. 3c).

\section{CLL cells with high ROR1 expression exhibit enhanced p62 accumulation and NRF2 activation}

CLL patients with leukemic cells expressing high levels of ROR1 exhibit enhanced disease-progression and shorter overall survival relative to ROR1-low patients [22]. We classified CLL cells based on ROR1 surface expression as ROR $1{ }^{\text {Lo }}$ or ROR $1{ }^{\mathrm{Hi}}$ CLL cells (Supplemental Table S1), as done previously [22]. We then compared BAFF receptors mRNA amounts in ROR $1^{\mathrm{Lo}}$ to ROR $1^{\mathrm{Hi}} \mathrm{CLL}$ cells. ROR $1^{\mathrm{Hi}}$ CLL cells showed significantly higher amounts of TNFRSF 17 mRNA, encoding the BAFF receptor BCMA, relative to ROR1 ${ }^{\text {Lo }}$ cells (Fig. 4a). Expression of TNFRSF $13 C$ and TNFRSC13B mRNAs, encoding BAFF-R and TACI, respectively, did not differ between the two cell populations (Fig. 4a). Relative to ROR1 ${ }^{\text {Lo }}$ CLL cells, ROR $1^{\mathrm{Hi}}$ CLL cells also expressed significantly higher amounts of p62 (Fig. 4b and Fig. S2a). Consistently, ROR $1^{\mathrm{Hi}}$ cells exhibited higher mRNA and protein expression of NRF2-target genes (Fig. 4c, d), suggesting a correlation between p62 expression and NRF2 activation. As expected from elevated SOD1 and NQO1 amounts, ROR $1^{\mathrm{Hi}}$ CLL cells exhibited lower mitochondrial ROS (mt-ROS) accumulation than ROR $1^{\text {Lo }}$ CLL cells (Fig. 4e), while no significant changes were detected in total cellular ROS 
Fig. 2 BAFF induces NF-кBdependent $p 62$ accumulation in CLL cells. a CLL cells were pretreated with $5 \mu \mathrm{M}$ BMS-

345541 for $1 \mathrm{hr}$ followed by $3 \mathrm{hr}$ incubation with $200 \mathrm{ng} / \mathrm{ml}$ BAFF. Nuclear phosphorylated p65 was analyzed by IB.

b Relative amounts of phosphorylated p65 in CLL cells from (a). c IB and d densitometric analysis of p62 in whole lysates of CLL cells treated as in (a). $n=3$. e IB analysis of lipidated LC3 (LC3II) in CLL cells pretreated with $10 \mu \mathrm{M}$ chloroquine (Chlorq) for $2 \mathrm{~h}$ and stimulated with BAFF for 3 and 6 hr. f Relative LC3-II amounts in CLL cells treated as in (e). Mean \pm sem. $n=5$. ${ }^{*} p<$ 0.05 ; ** $p<0.01$; *** $p<0.005$; $* * * * p<0.001$.

\section{a}

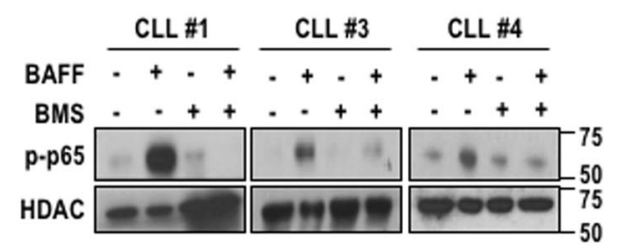

C

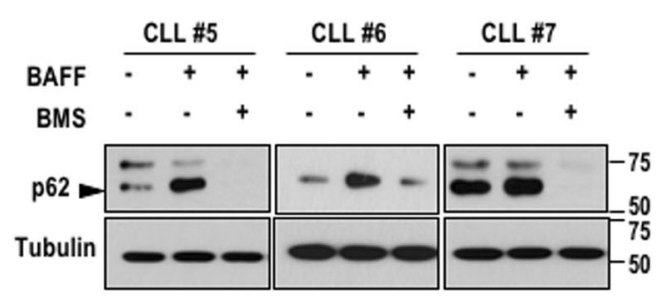

e
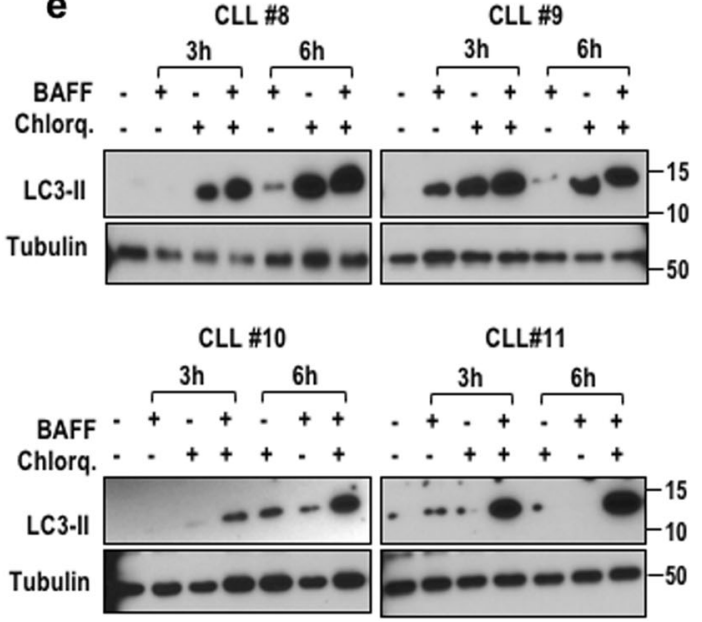

b

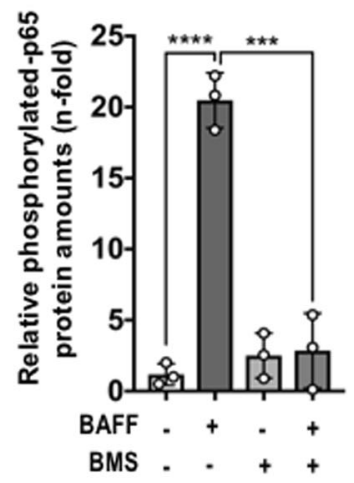

d

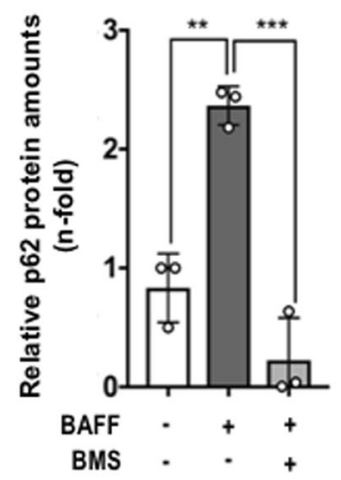

f

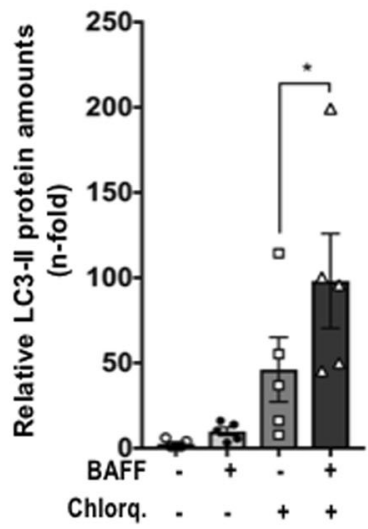

(Fig. S2b). Similarly, BAFF stimulation of CLL cells reduced mt-ROS accumulation (Fig. S2c, d). We also analyzed a published gene expression dataset, GSE13204 [23], designating CLL cells whose RORI mRNA amounts were above the median as ROR $1^{\text {High }}(n=224)$, and cells with RORI mRNA expression below the median as ROR $1^{\text {Low }}$ $(n=224)$. The NRF2 regulated SQSTM1, NQO1 and HMOX1 mRNAs were significantly more abundant in ROR $1^{\text {High }}$ CLL than in ROR $1^{\text {Low }}$ CLL cells (Fig. 4f). In addition, we performed gene expression analysis by comparing ROR $1^{>90 \%}(n=45)$, representing the top $10 \%$ of cells with highest RORI mRNA, to ROR $1^{<10 \%}(n=45)$, representing the bottom $10 \%$ of CLL cells in respect to
RORI mRNA. This analysis revealed that SQSTM1, NQOI and HMOXI were expressed at higher amounts in ROR $1^{>90 \%}$ cells relative to ROR $1^{<10 \%}$ cells (Fig. S2e). By gene set enrichment analysis (GSEA) we further examined whether NRF2 target genes [25], were enriched in ROR $1^{\text {High }}$ or ROR $1>90 \%$ CLL populations compared to ROR $1^{\text {Low }}$ or ROR $1<10 \%$ CLL. In both comparisons, GSEA revealed that NRF2 target genes were enriched in CLL cells with high RORI mRNA (Fig. 4g and Fig. S2f). Collectively, these data indicate that the p62-NRF2 cascade is upregulated in CLL cells with high expression of ROR1 and suggest that it may contribute to their improved survival and treatment resistance [22]. In addition to NRF2 target genes, 

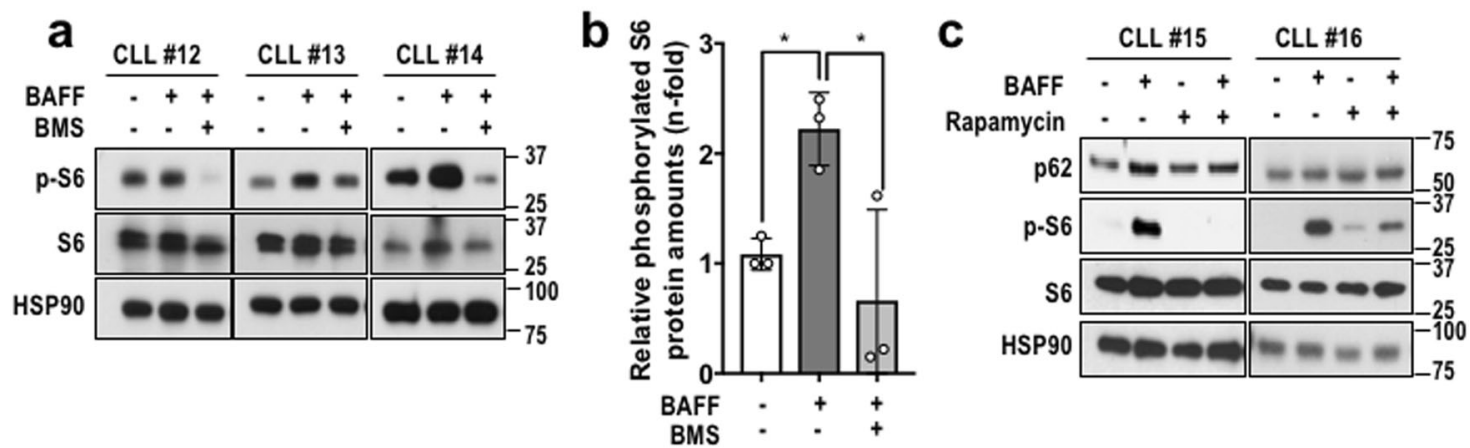

Fig. 3 BAFF leads to NF-кB-dependent mTORC1 activation. a CLL cells were pretreated with $5 \mu \mathrm{M}$ BMS-345541 for $1 \mathrm{hr}$ followed by $3 \mathrm{hr}$ BAFF treatment. Phosphorylated and total ribosomal S6 protein were examined by IB. Hsp90 was used as loading control. b Relative phosphorylated S6 amounts of CLL cells treated as in (a).
Mean \pm sem. $n=3$. ${ }^{*} p<0.05$. c CLL cells were pretreated with rapamycin for $1 \mathrm{hr}$ and then stimulated with BAFF as indicated. Presence of p62, non-phosphorylated and phosphorylated ribosomal S6 protein were analyzed by IB. the mTORC1 activation signature [26], was enriched in ROR $1^{\text {High }}$ relative to ROR $1^{\text {Low }}$ CLL cells (Fig. S2g, h). these results suggest a role for mTORC1 activation in ROR $1^{\text {High }}$ CLL cells.

Consistent with its ability to induce p62 accumulation, BAFF induced expression of the NRF2 target gene NQO1 in an NF-kB-dependent manner (Fig. 4h). Knockdown of p62 in MEC1-ROR1 cells confirmed that expression of NQO1 was indeed dependent on p62 (Fig. 4i).

\section{Cirmtuzumab reduces the NRF2 and mTORC1 signatures in CLL patients}

Cirmtuzumab, an antibody specific for ROR1, has shown activity in phase I clinical trials, reducing leukemic cell count and attenuating ROR1-dependent signaling [27]. Using GSEA we examined whether the NRF2 and/or mTORC1 signatures were altered in CLL cells isolated from patients treated with cirmtuzumab. CLL cells collected from patients before and 28 days after initiation of treatment were subjected to negative selection and were analyzed by RNAseq [27]. Cirmtuzumab treatment reduced mTORC1 induced genes (Fig. S3a), indicating that mTORC1 pathway is a downstream signaling of ROR1. Although cirmtuzumab treatment reduced the NRF2-target-gene-expression signature as well (Fig. 5a), we did not observe reduced expression of p62 or NQO1 in all samples examined (Fig. 5b), suggesting that the link between the p62-NRF2 cascade and ROR1 signaling in vivo may also be affected by other factors, such as BAFF.

\section{Targeting ROR1 ${ }^{\mathrm{Hi}} \mathrm{CLL}$ cells with NRF2-activatable prodrug}

ROR $1{ }^{\mathrm{Hi}}$ CLL cells expressed higher levels of NRF2-target genes, including $N Q O 1$, and maintained lower amounts of
mt-ROS in steady state than low ROR1-expressing cells (Fig. 4b-g). NRF2-signaling protects cancer cells from ROS toxicity [32], but systemic treatment with NRF2 inhibitors may result in undesired collateral toxicity. We therefore took advantage of elevated NRF2 activity and NQO1 expression in CLL cells to test their sensitivity to bioreductive prodrugs that are specially activated by NQO1, such as the oridonin derivative compound $29 \mathrm{~h}$ [21]. Compound 29h exhibits potent cytotoxic activity only against cells that express high amounts of NQO1, which is required for its metabolic activation [21]. Accordingly, ROR $1^{\mathrm{Hi}}$ CLL cells with abundant NQO1 expression were more susceptible to killing by compound 29h compared to low ROR1 or NQO1 expressing CLL cells (Fig. 6a and Fig. S4a). Treatment of NQO1-high CLL cells with compound 29h, resulted in PARP cleavage and accumulation of cytoplasmic cytochrome $\mathrm{C}$, both of which suggest activation of apoptosis (Fig. 6b). We also examined whether expression of NQO1 influenced the sensitivity of CLL cells to the ROS-inducing drug, venetoclax [33, 34]. We found that knockdown of NQO1 or p62 in MEC1-ROR1 CLL cells increased the cytotoxic activity of venetoclax (Fig. 6c and Fig. S4b). Combined treatment with compound $29 \mathrm{~h}$ and venetoclax resulted in greater cytotoxicity for high-NQO1-expressing CLL cells than treatment with either agent alone (Fig. 6d-f). Combination of compound $29 \mathrm{~h}$ with venetoclax also increased the accumulation of mt-ROS (Fig. 6g). Pretreatment with antioxidants reduced oxidative stress (Fig. S4c, d) but did not completely rescue cell viability (Fig. 6h).

\section{Discussion}

NF- $\mathrm{KB}$ activation is a common feature of CLL and other leukemias that accounts for enhanced proliferative activity 

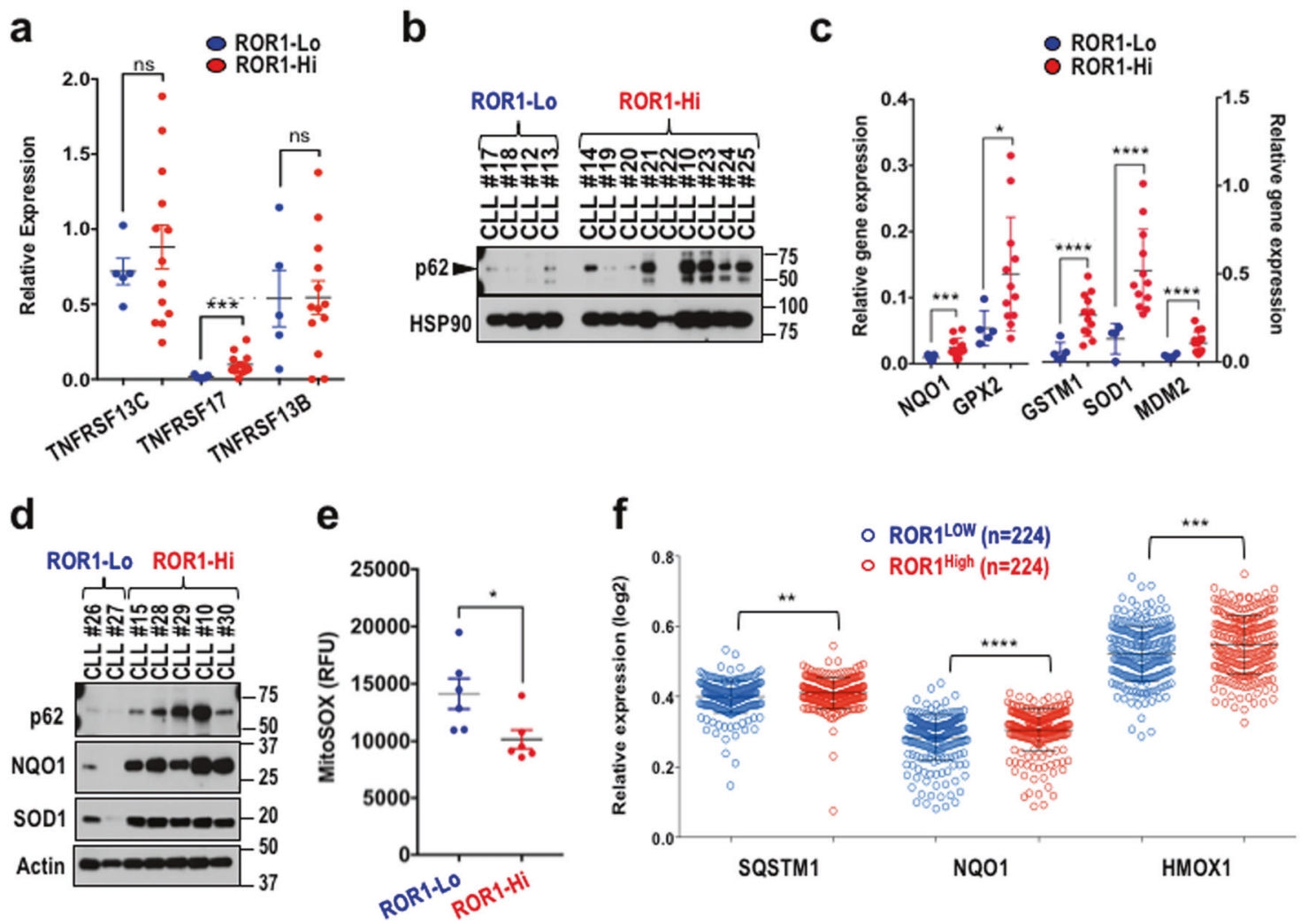
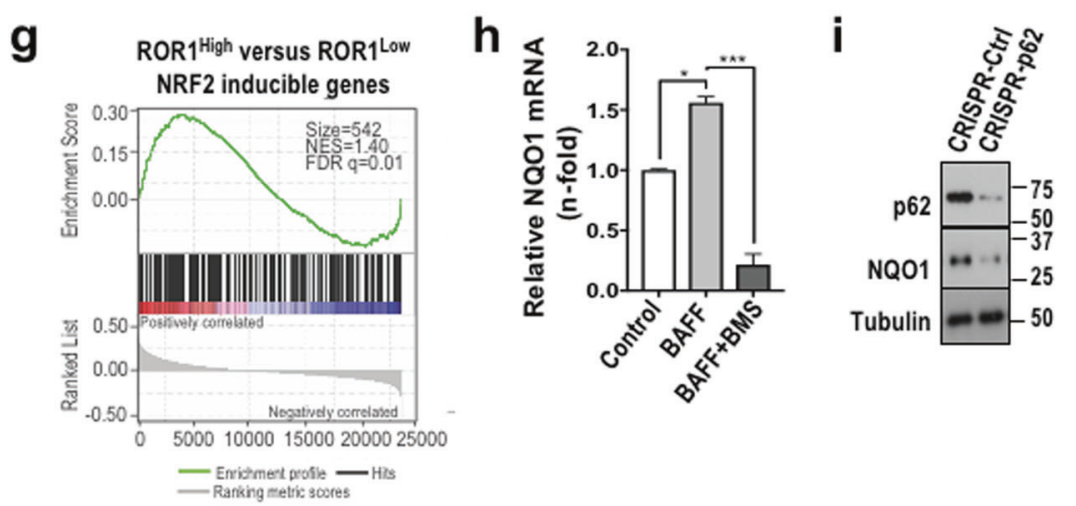

Fig. 4 NRF2 signaling is upregulated in CLL cells with high ROR1 expression. a mRNA expression of the TNFRSF13C, TNFRSF17, TNFRSF13B genes which code for BAFF-R, BCMA and TACI, respectively, was determined by QPCR in $\operatorname{ROR}^{\mathrm{Hi}}(n=13)$ and ROR ${ }^{\text {Lo }}$ CLL cells $(n=6)$. Mean \pm sem. b Representative IB showing p62 expression in ROR $1^{\mathrm{Hi}}$ and ROR $1^{\mathrm{Lo}}$ CLL cells. c Expression of NQO1, GPX2, GSTM1, SOD1, MDM2 mRNAs in ROR1 ${ }^{\mathrm{Hi}}(n=13)$ and ROR $1^{\text {Lo }}(n=6)$ CLL cells analyzed by QPCR. Mean \pm sem. d p62, NQO1, SOD1 expression in ROR $1^{\mathrm{Hi}}$ and ROR $1^{\text {Lo }}$ CLL cells was determined by IB analysis. e MitoSOX relative fluorescence in ROR $1^{\mathrm{Hi}}$ versus ROR $1^{\mathrm{Lo}}$ CLL cases. Mean \pm sem. $n=6$. f SQSTM1,

and reduced apoptosis [35]. In contrast to other leukemias, recurrent mutations that result in active $\mathrm{NF}-\mathrm{\kappa B}$ signaling are quite rare in CLL and by-and-large NF- $\mathrm{\kappa B}$ in CLL is activated in response to microenvironmental factors, such as BAFF [7]. Regardless of the mechanism responsible for
$N Q O 1$, and $H M O X 1 \mathrm{mRNA} \log 2$ relative expression data from 224 ROR $1^{\text {High }}$ CLL cases and 224 ROR $1^{\text {Low }}$ CLL cases as $\log 2$ relative expression. g GSEA plot of NRF2-inducible genes on microarray data available in GEO database (GSE13204) comparing ROR $1^{\text {High }}$ versus ROR $1^{\text {Low }}$ CLL cases. Size, NES and FDR $q$ value are as indicated. h CLL cells were pretreated with BMS-345541 for $1 \mathrm{hr}$ and stimulated with $200 \mathrm{ng} / \mathrm{ml}$ BAFF for $5 \mathrm{hr}$. NQO1 mRNA was examined by QPCR. Mean \pm sd. $n=2$ CLL cell samples. i p62 and NQO1 expression in CRISPR-Ctrl and CRISPR-p62 MEC1-ROR1 cells were detected by IB. $* p<0.05$; ** $p<0.01 ; * * * p<0.005 ; * * * * p<0.001$; ns, not significant.

NF- $\mathrm{KB}$ activation, our understanding of the effectors through which it contributes to the malignant phenotype of CLL is incomplete. The results described in this study indicate that the signaling adaptor p62/SQSTM1 provides an important link between NF- $\mathrm{kB}$ and another prominent 
Fig. 5 Cirmtuzumab inhibits NRF2 signaling in vivo. a GSEA plot of NRF2-inducible genes on the transcriptome of three paired CLL samples preand at day 28 (D28) postcirmtuzumab treatment. Size, NES and FDR $q$ value are as indicated. b IB analysis of p62 and NQO1 in three CLL cases pre- and at D28 postcirmtuzumab treatment.
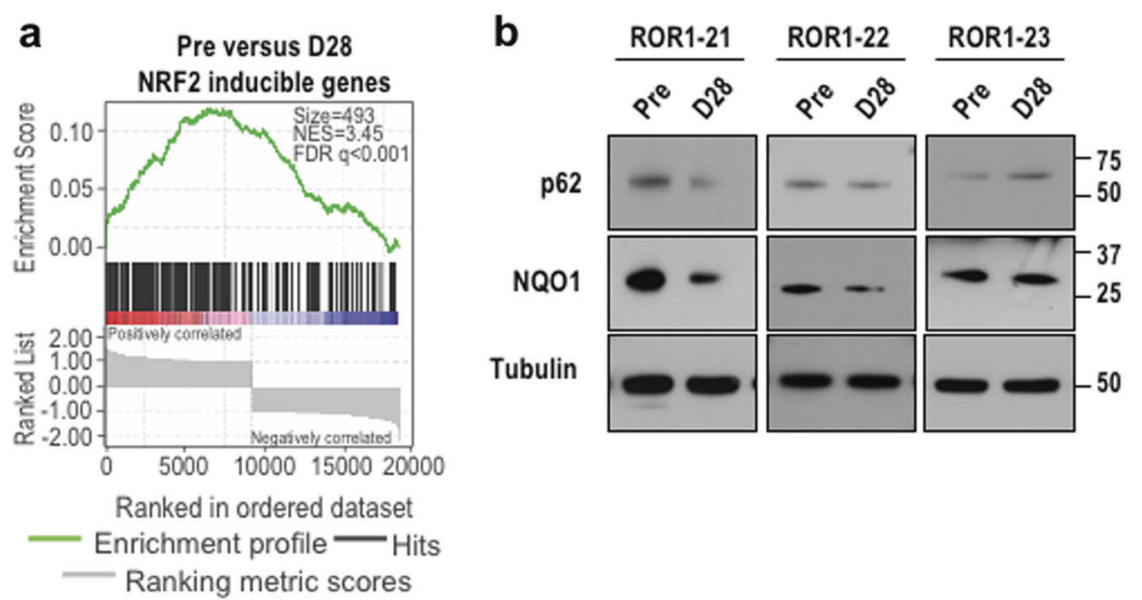

transcription factor NRF2, which was recently found to be activated in various solid-tissue malignancies [16, 36-39]. In addition to its ability to re-wire tumor cell metabolism [40], NRF2 protects cancer cells from drug-induced oxidative stress by inducing expression of ROS detoxifying enzymes [41-43]. In CLL cells, p62-mediated NRF2 activation also contributes to drug resistance, especially in ROR $1^{\mathrm{Hi}}$ CLL cells, which express considerably higher amounts of p62 than ROR $1^{\text {Lo }}$ CLL cells. It should be noted, however, that NF- $\mathrm{KB}$ only controls expression of SQSTM1 mRNA, but the amount of p62 expressed by any given cell depends on other factors, specially the rate of autophagy, through which p62 is degraded [11]. Interestingly, BAFF treatment enhanced rather than inhibited autophagic flux, suggesting that its ability to induce p62 accumulation is mainly dependent on NF- $\kappa \mathrm{B}$ activation. Recent studies have shown elevated expression of p62 in various solid malignancies, including hepatocellular carcinoma (HCC) and pancreatic ductal adenocarcinoma (PDAC), where p62 was found to be intimately involved in malignant progression and served as an important NRF2 activator [11, 16, 17]. Although we cannot rule out that other activators or molecules related with enhanced $\mathrm{NF}-\kappa \mathrm{B}$ signaling play a part in p62-NRF2 cascade activation, we found that expression of p62 could be induced by receptors for BAFF or other signaling receptors, such as ROR1. Of note, consistent with activation of NRF2 signaling and a more efficient antioxidant response, both ROR $1^{\mathrm{Hi}}$ CLL cells and CLL cells stimulated with BAFF exhibited lower mt-ROS amounts in steady state than ROR $1^{\text {Lo }}$ CLL cells or unstimulated cells respectively.

Once expressed in sufficiently high amounts, p62 drives activation of NRF2 through the sequestration of KEAP1 [11], and potentiates mTORC1 activation, a signaling pathway that can be blocked by the ROR1 antibody cirmtuzumab. Although the contribution of mTORC1 to
NRF2 activation and the CLL malignant phenotype remains to be determined, our results show that NRF2 activation may confer a drug-resistant phenotype in part through induction of NQO1, whose ablation enhanced the susceptibility of CLL cells to killing by the ROS-producing BCL2-inhibitor venetoclax. However, elevated expression of NQO1 also renders ROR $1^{\mathrm{Hi}}$ CLL cells more sensitive to compound $29 \mathrm{~h}$, a prodrug that is activated by an NQO1-dependent mechanism [21]. Importantly, the combination of compound $29 \mathrm{~h}$ with venetoclax, not only potentiates oxidative stress in ROR $1^{\mathrm{Hi}}$ CLL cells but also reduces their viability. Therefore, we suggest that elevated NQO1 expression may represent an "Achilles Heel" of ROR $1{ }^{\mathrm{Hi}}$ CLL cells, which are generally more aggressive than ROR $1^{\text {Lo }}$ CLL cells [22]. Of note, CLL treatment with two doses of $16 \mathrm{mg} / \mathrm{kg}$ cirmtuzumab reduces expression of p62 and NQO1 only in some patients, suggesting that some ROR1 Hi CLL cells may also be stimulated by factors other than ROR1, for instance BAFF which sustains p62- and NRF2signaling independent of ROR1. In a similar vein, activation of $\mathrm{NF}-\kappa \mathrm{B}$ signaling by exposure to $\mathrm{BAFF}$ or TNF increases survival of mantle cell lymphoma cells even when treated with another ROR1 monoclonal antibody 2A2, which also inhibits Wnt5a-ROR1 signaling in ROR1 ${ }^{+}$neoplastic cells [44], acting like cirmtuzumab [27, 45]. Conceivably, compound $29 \mathrm{~h}$ could be used in combination with cirmtuzumab or other anti-ROR 1 antibodies to selectively target ROR $1^{\mathrm{Hi}}$ CLL cells.

In summary, in this study we revealed that activation of the NF- $\kappa \mathrm{B}$ dependent p62-NRF2 cascade contributes to the survival and therapy resistance of CLL cells that express high-levels of ROR1 and that treatment with cirmtuzumab, alone or in combination with compound $29 \mathrm{~h}$, could target ROR $1^{\mathrm{Hi}}$ CLL cells and enhance their susceptibility to venetoclax therapy (Fig. 6i). 
a

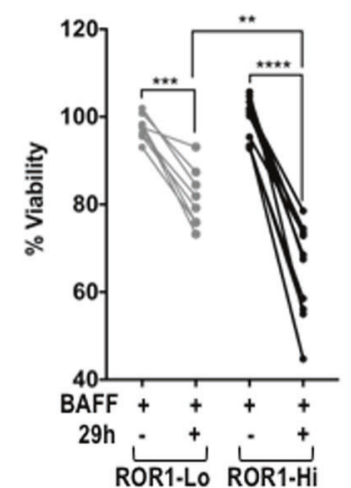

b

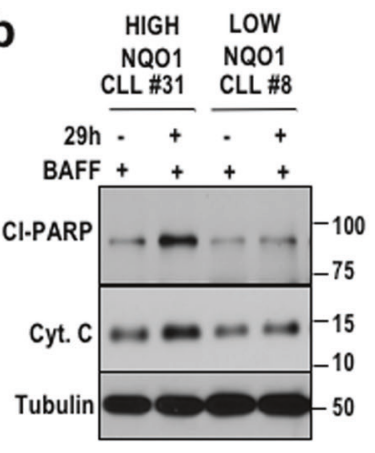

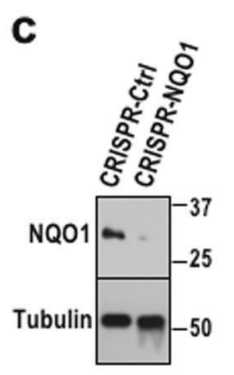

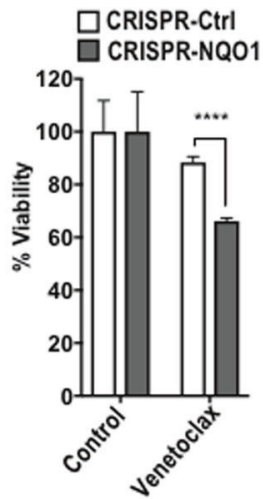

d

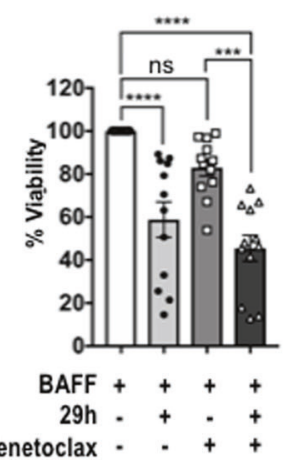

e

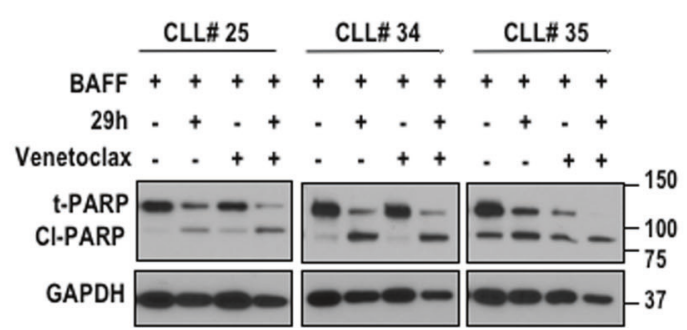

f

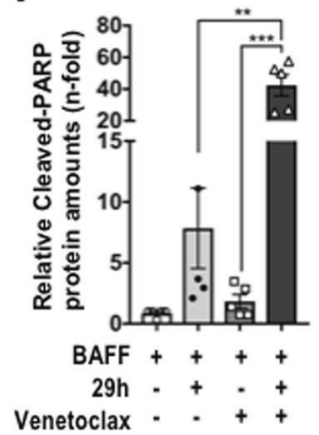

g

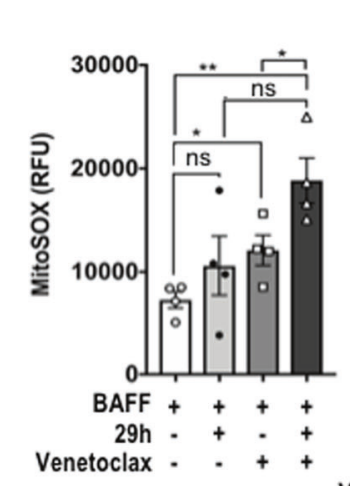

h

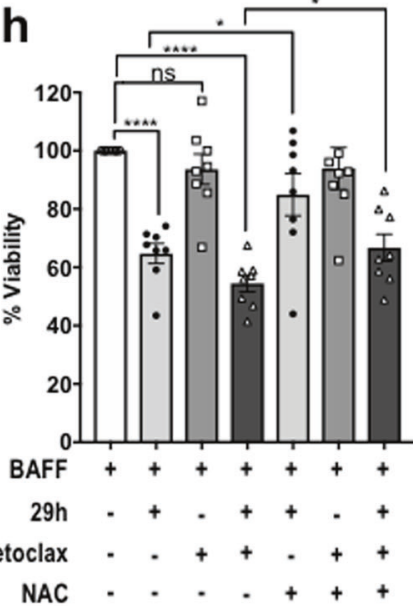

i

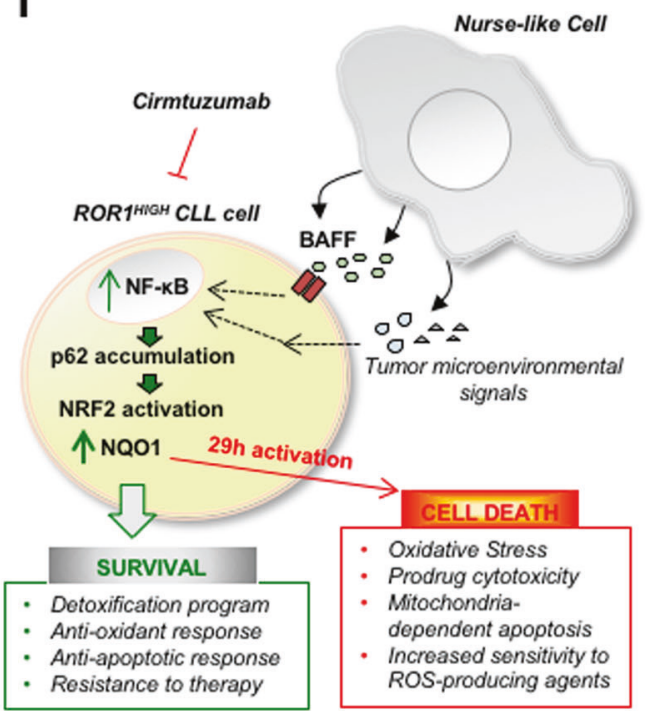

Fig. 6 NRF2 activation protects CLL cells that express high ROR1 from the ROS-inducing drug venetoclax. a Relative viability of ROR $1^{\mathrm{Hi}}$ and ROR $1^{\mathrm{Lo}}$ CLL cells pretreated with BAFF for $1 \mathrm{hr}$ and treated with $0.25 \mu \mathrm{M} 29 \mathrm{~h}$ for $24 \mathrm{hr}$. b IB analysis of cleaved-PARP (Cl-PARP) and cytoplasmic cytochrome C (Cyt. C) in CLL cells that are either NQO1 high or low and treated as in (a). c Relative viability of CRISPR-Ctrl and CRISPR-NQO1 MEC1-ROR1 cells treated with $1 \mathrm{nM}$ venetoclax for $48 \mathrm{hr}$. d Relative viability of ROR $1^{\mathrm{Hi}} \mathrm{CLL}$ cells stimulated with BAFF for $1 \mathrm{hr}$ and then treated with $1 \mathrm{nM}$ venetoclax and $0.25 \mu \mathrm{M} 29 \mathrm{~h}$ either alone or in combination for $36 \mathrm{hr}$. $n=12$. e IB

Acknowledgements We thank eBioscience, Cell Signaling Technologies, Santa Cruz Technologies and Promega for gifts of reagents. We also thank Christina Dooka, Xuemei Piao, Monica Spydell and Tuan analysis of cleaved PARP (Cl-PARP) and total PARP (t-PARP) in ROR $1^{\mathrm{Hi}}$ CLL cells treated as in (d). f Relative cleavage of PARP in ROR $1^{\mathrm{Hi}}$ CLL cells treated as in (d). $n=5$. g MitoSOX relative fluorescence in ROR $1{ }^{\mathrm{Hi}}$ CLL cells treated as in (d). $n=4$. h Relative viability of $\mathrm{ROR} 1^{\mathrm{Hi}}$ CLL cells pretreated with $1 \mu \mathrm{M}$-acetyl L-cysteine (NAC) for $1 \mathrm{hr}$ and then stimulated with BAFF and treated with $1 \mathrm{nM}$ venetoclax and $0.25 \mu \mathrm{M}$ compound $29 \mathrm{~h}$ for $36 \mathrm{hr} . n=8$. $* p<0.05 ; * * p<0.01 ; * * * p<0.005 ; * * * *<<0.001$; ns, not significant. i Schematic working model summarizing our results.

Tran for their excellent technical assistance. This work was supported by SCOR grant (7005-14) from the Leukemia and Lymphoma Society to TJK and MK; NIH (R01AI43477 and R37AI043477), NIEHS 
Superfund basic research program (P42ES010337), The Rotary Coins for Alzheimer's Research Trust Fund (CART Fund) awards to MK, who is an American Cancer Research Society Professor and holds the Ben and Wanda Hildyard Chair for Mitochondrial and Metabolic Disease. This work was also supported by grants R37-CA049870 and R01-CA236361 to TJK. ES-L was supported by Sara Borrell fellowship from ISCIII/MICINN. EMG was supported by the Blood Cancer research Fund. LA was supported by the International Cancer Research Fellowship (iCARE) and AIRC co-founded by the European Union, and a C3 Pedal the Cause grant.

Author contributions ES-L, EMG, TJK, and MK conceived the project. ES-L and EMG designed and performed most of the experiments. LA and NS provided assistance with experiments and analyses. LZR provided and characterized primary CLL cells. JX and BS designed and provided prodrug compound $29 \mathrm{~h}$. LA., LZR, TJK, and MK provided advice. TJK and MK supervised the project. ES-L, EMG, TJK, and MK wrote the manuscript with input from all authors.

\section{Compliance with ethical standards}

Conflict of interest Cirmtuzumab was developed in the Kipps laboratory and licensed by the University of California, San Diego to Oncternal, which has provided stock/options to the university and to TJK.

Publisher's note Springer Nature remains neutral with regard to jurisdictional claims in published maps and institutional affiliations.

\section{References}

1. Kipps TJ, Stevenson FK, Wu CJ, Croce CM, Packham G, Wierda WG, et al. Chronic lymphocytic leukaemia. Nat Rev Dis Prim. 2017;3:17008.

2. Burger JA, Tsukada N, Burger M, Zvaifler NJ, Dell'Aquila M, Kipps TJ. Blood-derived nurse-like cells protect chronic lymphocytic leukemia B cells from spontaneous apoptosis through stromal cell-derived factor-1. Blood. 2000;96:2655-63.

3. Novak AJ, Bram RJ, Kay NE, Jelinek DF. Aberrant expression of B-lymphocyte stimulator by B chronic lymphocytic leukemia cells: a mechanism for survival. Blood. 2002;100:2973-9.

4. Haiat S, Billard C, Quiney C, Ajchenbaum-Cymbalista F, Kolb JP. Role of BAFF and APRIL in human B-cell chronic lymphocytic leukaemia. Immunology. 2006;118:281-92.

5. Sanchez E, Tanenbaum EJ, Patil S, Li M, Soof CM, Vidisheva $\mathrm{A}$, et al. The clinical significance of B-cell maturation antigen as a therapeutic target and biomarker. Expert Rev Mol Diagn. 2018;18:319-29.

6. McWilliams EM, Lucas CR, Chen T, Harrington BK, Wasmuth R, Campbell A, et al. Anti-BAFF-R antibody VAY-736 demonstrates promising preclinical activity in CLL and enhances effectiveness of ibrutinib. Blood Adv. 2019;3:447-60.

7. Endo T, Nishio M, Enzler T, Cottam HB, Fukuda T, James DF, et al. BAFF and APRIL support chronic lymphocytic leukemia Bcell survival through activation of the canonical NF-kappaB pathway. Blood. 2007;109:703-10.

8. Zhang W, Kater AP, Widhopf GF 2nd, Chuang HY, Enzler T, James DF, et al. B-cell activating factor and v-Myc myelocytomatosis viral oncogene homolog (c-Myc) influence progression of chronic lymphocytic leukemia. Proc Natl Acad Sci USA. 2010; 107:18956-60.

9. Mansouri L, Papakonstantinou N, Ntoufa S, Stamatopoulos K, Rosenquist R. NF-kappaB activation in chronic lymphocytic leukemia: a point of convergence of external triggers and intrinsic lesions. Semin Cancer Biol. 2016;39:40-8.

10. Greten FR, Arkan MC, Bollrath J, Hsu LC, Goode J, Miething C, et al. NF-kappaB is a negative regulator of IL-1beta secretion as revealed by genetic and pharmacological inhibition of IKKbeta. Cell. 2007;130:918-31.

11. Moscat J, Karin M, Diaz-Meco MT. p62 in Cancer: Signaling Adaptor Beyond Autophagy. Cell. 2016;167:606-9.

12. Linares JF, Duran A, Yajima T, Pasparakis M, Moscat J, DiazMeco MT. K63 polyubiquitination and activation of mTOR by the p62-TRAF6 complex in nutrient-activated cells. Mol Cell. 2013;51:283-96.

13. Komatsu M, Kurokawa H, Waguri S, Taguchi K, Kobayashi A, Ichimura $\mathrm{Y}$, et al. The selective autophagy substrate p62 activates the stress responsive transcription factor $\mathrm{Nrf} 2$ through inactivation of Keap1. Nat Cell Biol. 2010;12:213-23.

14. Zhong Z, Umemura A, Sanchez-Lopez E, Liang S, Shalapour S, Wong J, et al. NF-kappaB restricts inflammasome activation via elimination of damaged mitochondria. Cell. 2016;164:896-910.

15. Hsu LC, Enzler T, Seita J, Timmer AM, Lee CY, Lai TY, et al. IL-1beta-driven neutrophilia preserves antibacterial defense in the absence of the kinase IKKbeta. Nat Immunol. 2011;12:144-50.

16. Todoric J, Antonucci L, Di Caro G, Li N, Wu X, Lytle NK, et al. Stress-activated NRF2-MDM2 cascade controls neoplastic progression in pancreas. Cancer Cell. 2017;32:824-39.e8.

17. Umemura A, He F, Taniguchi $K$, Nakagawa $H$, Yamachika $S$, Font-Burgada J, et al. p62, Upregulated during preneoplasia, induces hepatocellular carcinogenesis by maintaining survival of stressed HCC-initiating cells. Cancer Cell. 2016;29:935-48.

18. Tsukada N, Burger JA, Zvaifler NJ, Kipps TJ. Distinctive features of "nurselike" cells that differentiate in the context of chronic lymphocytic leukemia. Blood. 2002;99:1030-7.

19. Yu J, Chen L, Chen Y, Hasan MK, Ghia EM, Zhang L, et al. Wnt5a induces ROR1 to associate with 14-3-3zeta for enhanced chemotaxis and proliferation of chronic lymphocytic leukemia cells. Leukemia. 2017;31:2608-14.

20. Burke JR, Pattoli MA, Gregor KR, Brassil PJ, MacMaster JF, McIntyre KW, et al. BMS-345541 is a highly selective inhibitor of I kappa B kinase that binds at an allosteric site of the enzyme and blocks NF-kappa B-dependent transcription in mice. J Biol Chem. 2003;278:1450-6.

21. Xu S, Yao H, Pei L, Hu M, Li D, Qiu Y, et al. Design, synthesis, and biological evaluation of $\mathrm{NAD}(\mathrm{P}) \mathrm{H}$ : Quinone oxidoreductase (NQO1)-targeted oridonin prodrugs possessing indolequinone moiety for hypoxia-selective activation. Eur $\mathrm{J}$ Med Chem. 2017;132:310-21.

22. Cui B, Ghia EM, Chen L, Rassenti LZ, DeBoever C, Widhopf GF 2nd, et al. High-level ROR1 associates with accelerated disease progression in chronic lymphocytic leukemia. Blood. 2016;128:2931-40

23. Kohlmann A, Kipps TJ, Rassenti LZ, Downing JR, Shurtleff SA, Mills KI, et al. An international standardization programme towards the application of gene expression profiling in routine leukaemia diagnostics: the Microarray Innovations in LEukemia study prephase. Br J Haematol. 2008;142:802-7.

24. Subramanian A, Tamayo P, Mootha VK, Mukherjee S, Ebert BL, Gillette MA, et al. Gene set enrichment analysis: a knowledgebased approach for interpreting genome-wide expression profiles. Proc Natl Acad Sci USA. 2005;102:15545-50.

25. Malhotra D, Portales-Casamar E, Singh A, Srivastava S, Arenillas D, Happel C, et al. Global mapping of binding sites for Nrf2 identifies novel targets in cell survival response through ChIP-Seq profiling and network analysis. Nucleic Acids Res. 2010;38:5718-34. 
26. Duvel K, Yecies JL, Menon S, Raman P, Lipovsky AI, Souza AL, et al. Activation of a metabolic gene regulatory network downstream of mTOR complex 1. Mol Cell. 2010;39:171-83.

27. Choi MY, Widhopf GF 2nd, Ghia EM, Kidwell RL, Hasan MK, $\mathrm{Yu}$ J, et al. Phase I trial: cirmtuzumab inhibits ror1 signaling and stemness signatures in patients with chronic lymphocytic leukemia. Cell Stem Cell. 2018;22:951-9. e3.

28. Nishio M, Endo T, Tsukada N, Ohata J, Kitada S, Reed JC, et al. Nurselike cells express BAFF and APRIL, which can promote survival of chronic lymphocytic leukemia cells via a paracrine pathway distinct from that of SDF-1alpha. Blood. 2005; 106:1012-20.

29. Antonucci L, Fagman JB, Kim JY, Todoric J, Gukovsky I, Mackey M, et al. Basal autophagy maintains pancreatic acinar cell homeostasis and protein synthesis and prevents ER stress. Proc Natl Acad Sci USA. 2015;112:E6166-74.

30. Duran A, Amanchy R, Linares JF, Joshi J, Abu-Baker S, Porollo A, et al. p62 is a key regulator of nutrient sensing in the mTORC1 pathway. Mol Cell. 2011;44:134-46.

31. Hay N, Sonenberg N. Upstream and downstream of mTOR. Genes Dev. 2004;18:1926-45.

32. Arlt A, Bauer I, Schafmayer C, Tepel J, Muerkoster SS, Brosch $\mathrm{M}$, et al. Increased proteasome subunit protein expression and proteasome activity in colon cancer relate to an enhanced activation of nuclear factor E2-related factor 2 (Nrf2). Oncogene. 2009;28:3983-96.

33. Aharoni-Simon M, Shumiatcher R, Yeung A, Shih AZ, Dolinsky VW, Doucette CA, et al. Bcl-2 regulates reactive oxygen species signaling and a redox-sensitive mitochondrial proton leak in mouse pancreatic beta-cells. Endocrinology. 2016;157:2270-81.

34. Xiong L, Tang Y, Liu Z, Dai J, Wang X. BCL-2 inhibition impairs mitochondrial function and targets oral tongue squamous cell carcinoma. Springerplus. 2016;5:1626.

35. DiDonato JA, Mercurio F, Karin M. NF-kappaB and the link between inflammation and cancer. Immunol Rev. 2012;246: 379-400.
36. DeNicola GM, Chen PH, Mullarky E, Sudderth JA, Hu Z, Wu D, et al. NRF2 regulates serine biosynthesis in non-small cell lung cancer. Nat Genet. 2015;47:1475-81.

37. Zhang M, Zhang C, Zhang L, Yang Q, Zhou S, Wen Q, et al. Nrf2 is a potential prognostic marker and promotes proliferation and invasion in human hepatocellular carcinoma. BMC Cancer. 2015;15:531.

38. Wu RP, Hayashi T, Cottam HB, Jin G, Yao S, Wu CC, et al. Nrf2 responses and the therapeutic selectivity of electrophilic compounds in chronic lymphocytic leukemia. Proc Natl Acad Sci USA. 2010;107:7479-84.

39. Saito T, Ichimura Y, Taguchi K, Suzuki T, Mizushima T, Takagi $\mathrm{K}$, et al. p62/Sqstm 1 promotes malignancy of HCV-positive hepatocellular carcinoma through Nrf2-dependent metabolic reprogramming. Nat Commun. 2016;7:12030.

40. Mitsuishi Y, Taguchi K, Kawatani Y, Shibata T, Nukiwa T, Aburatani $\mathrm{H}$, et al. Nrf2 redirects glucose and glutamine into anabolic pathways in metabolic reprogramming. Cancer Cell. 2012;22:66-79.

41. Lin P, Ren Y, Yan X, Luo Y, Zhang H, Kesarwani M, et al. The high NRF2 expression confers chemotherapy resistance partly through up-regulated DUSP1 in myelodysplastic syndromes. Haematologica. 2019;104:485-96.

42. Wang XJ, Sun Z, Villeneuve NF, Zhang S, Zhao F, Li Y, et al. Nrf2 enhances resistance of cancer cells to chemotherapeutic drugs, the dark side of Nrf2. Carcinogenesis. 2008;29:1235-43.

43. Karathedath S, Rajamani BM, Musheer Aalam SM, Abraham A, Varatharajan S, Krishnamurthy $\mathrm{P}$, et al. Role of NF-E2 related factor 2 (Nrf2) on chemotherapy resistance in acute myeloid leukemia (AML) and the effect of pharmacological inhibition of Nrf2. PLoS ONE. 2017;12:e0177227.

44. Karvonen H, Chiron D, Niininen W, Ek S, Jerkeman M, Moradi E, et al. Crosstalk between ROR1 and BCR pathways defines novel treatment strategies in mantle cell lymphoma. Blood Adv. 2017;1:2257-68.

45. Yu J, Chen L, Cui B, Wu C, Choi MY, Chen Y, et al. Cirmtuzumab inhibits Wnt5a-induced Rac1 activation in chronic lymphocytic leukemia treated with ibrutinib. Leukemia. 2017;31:1333-9. 